\title{
Model of Evaluating the Effectiveness of the Information Technology for the Tasks of the Thermophysical Properties of Substances
}

\author{
Sura Khalil Ibrahim \\ Al Nisour University College \\ Department of Computer Engineering Techniques \\ Baghdad, Iraq
}

\begin{abstract}
Specialized commercial software packages have been developed and used in the world, which make it possible to obtain data on the thermophysical properties for certain classes of substances and materials. The experience of the practical use of such programs revealed their significant shortcomings. They do not provide access to the tables of primary experimental data, do not allow to select models for their processing, the models used are not physically sound, which does not allow extrapolation of data, there is no real possibility to predict properties, etc. In Russia, this direction has not yet received a noticeable spread. The Institute of Computational Technologies of the Siberian Branch of the Russian Academy of Sciences in cooperation with the Institute of Thermal Physics of the Siberian Branch of the Russian Academy of Sciences have developed the Information Computing System http://tick2.ict.sbras.ru/, which will allow to combine the advantages of existing codes and eliminate the above disadvantages. The created resource will become an important tool for the development of theories of substance properties and phase transformations.
\end{abstract}

\section{Keywords}

web-based data processing, data analysis, semantic model, properties of substances, phase transformations.

\section{INTRODUCTION}

Information technologies have a huge impact on all areas of human activity related to the accumulation and processing of information. In a relatively short time, the existence of information and communication technologies has accumulated a huge amount of diverse data presented exclusively in electronic form. There are problems of providing access (including remote) of users to heterogeneous types and data formats, processing and interpreting the results of observations of the properties of substances and phase transformations.Integration of information resources into a single information environment and organization of access to computing resources is one of the most important areas for the development of modern information technologies. Solving the problems of creating and integrating information resources and products is becoming a prerequisite for the development of many countries, including Russia. The rapid development of global information and computing networks leads to a change in the fundamental paradigms of data processing, which can be characterized as a transition to the support and development of distributed information and computing resources [1]. Technologies for using distributed information and computing resources are gaining increasing priority in the information society. At the same time, there is a transition to an exclusively distributed scheme for creating, maintaining, storing resources 1 and striving for virtual unity by providing free access to any network resources through a limited number of access points. The principle of forming a single, mathematically uniform field of computer information in the network resources is postulated, which can become a universal and machine-independent data carrier, unified programs and globally distributed computing processes.

\section{SUBJECT AREA}

The study of the behavior of thermophysical properties of thermodynamic systems consisting of a large number of strongly interacting particles is one of the most important fundamental problems of modern physics and chemistry. The solution of the corresponding problems from the "first principles" is currently impossible due to the general incompleteness of knowledge, insufficient adequacy of the models and enormous mathematical difficulties in using them. In this situation, the construction of new physical models that would allow describing the behavior of such systems in a wide range of state parameters, including areas of phase transformations, is possible only on the basis of reliable experimental data. They are also the basis for the development of methods for the reliable assessment of properties based on a limited amount of experimental information. Such studies are possible only with reliable, thermodynamically consistent data and the possibility of analyzing them with arbitrary state parameters, including lines of phase equilibria. This approach, in addition to performing experimental studies, involves conducting an expert assessment of the existing array of measurement results with a reasonable choice of recommended values and an assessment of their errors, processing heterogeneous data using physically sound and thermodynamically consistent models, and, most importantly, building information and analytical systems which will allow to quickly obtain data on the properties of substances at arbitrary values of temperature, pressure and composition.

A wide range of experimental facilities allows for studies of melting points, structural transitions, liquids, solidus, liquidliquid equilibrium, etc. The data of experimental studies and data of researchers from other research groups are presented in the literature (articles, reference books). They are significant in volume and variety. This circumstance makes it necessary and relevant to use distributed information and computing systems for the above tasks. The use of electronic versions provides the user with on-line access to the results of observations, storage and mathematical processing. 


\section{EXISTING INFORMATION SYSTEMS}

Such a task can be solved by a specialized problem-oriented Information System, which will allow solving the problem of storing this empirical material, its processing using modern mathematical algorithms. This will allow transferring work with these data to a qualitatively higher level, opening up prospects for the formulation and effective solution of new scientific and practical problems. The development of databases on the thermophysical properties of substances and materials was solved by many research teams, both in Russia and abroad. Specialized commercial software packages are known that allow one to obtain data on the thermophysical properties for certain classes of substances and materials. In Russia, this direction has not yet received a noticeable spread. It should be noted that the experience of the practical use of such programs revealed their significant shortcomings. They do not provide access to the tables of primary experimental data, do not allow to select models for their processing, the models used are not physically sound, which does not allow extrapolation of data, there is no real possibility to predict properties, etc.The National Institute of Standards and Technology (NIST) has developed the "NIST Chemistry WebBook" resource at http://webbook.nist.gov/. It provides access to data collected and distributed by NIST through a standard reference data program.

\section{NIST Chemistry WebBook contains:}

- Thermochemical data for more than 7000 organic and inorganic and non-organic compounds: Enthalpy of formation, enthalpy of combustion, heat capacity, entropy, phase transition values of enthalpy and temperature, vapor pressure.

- Thermochemistry reaction data for more than 8000 reactions.

- IR spectra for more than 16,000 compounds.

- Mass spectra for over 33,000 compounds.

- UV / Vis spectra of more than 1600 compounds.

- Gas chromatography data, for more than 27,000 compounds.

- Electronic and vibrational spectra of more than 5000 compounds.

- Constants of diatomic molecules (spectroscopic data) for more than 600 compounds.

- Ion energetics data, for more than 16,000 compounds including: ionization energy, energy appearance, electron affinity, proton affinity, basicity gas and cluster ion binding energy.

- Thermophysical properties of data for 74 fluids, namely: density, specific volume, heat capacity at constant pressure $\left(\mathrm{C}_{\mathrm{p}}\right)$, heat capacity at constant volume $(\mathrm{CV})$, enthalpy, internal energy, entropy, viscosity, thermal conductivity, Joule-Thomson coefficient, surface tension (saturation curve only), sound velocity. Here you can find information about specific compounds in WebBook chemistry based on name, chemical formula, CAS registration number, molecular weight, chemical structure, or selected ion energies and spectral properties.

Information system for heat supply [4] http://www.rosteplo.ru/soft/1/ was created by the non-profit partnership "Russian Heat Supply". It seems that ideologically, the "Thermophysical Properties of Substances" section could be the most interesting for us, one of the components of which is the Electronic Reference Book "Thermophysical Properties of Heat Transfer Fluids". It allows the calculation of thermal properties (specific volume, enthalpy, entropy, heat capacity, thermal conductivity, etc. The portal [5] "THERMOPHYSICS.RU", http://www.thermophysics.ru/modules.php?name=News\&file $=$ article $\&$ sid $=4$, is widely known by specialists. It provides information space for scientific, informational and educational support of domestic thermal physicists. The subject of documents on the portal covers the main sections of thermal physics, including technical thermodynamics, thermal properties of substances and materials, heat and mass transfer, etc. The specific choice of resources and services is focused on the training of specialists in heat power engineering and heating engineering at the Russian Higher School and complies with the established traditions of teaching at the Moscow Power Engineering Institute. The portal was developed within the framework of the joint program "Integration of fundamental science and higher education" with the participation of JIHT RAS and the MEI and has been functioning since 2003. The development and creation of the system is carried out by the teams of the Institute of High Temperatures of the Russian Academy of Sciences.

Through the efforts of the teams of the Institute for High Temperatures of the Russian Academy of Sciences and the Moscow Power Engineering Institute, under the supervision of Prof. Dr. med. G.A. Kobzeva and Dr. of Sc. LR Fokina, was created, maintained and updated the resource http://thermophysics.ru/triptych/ - "The information triptych of thermophysical properties of substances: a guide on the Internet, a knowledge base and an electronic guide" [6]. In reality, this resource is an information and analytical system for storing and disseminating bibliographic and numerical data on the thermophysical properties of substances. It is an integrated environment combining the capabilities of three products:

- electronic reference book on thermophysical properties of substances for thermal power engineering;

- information system "EPIDIF" (knowledge base), which provides calculations of transport properties of rarefied gas mixtures;

- guide to the thermophysical resources of the Internet.

Data sources are bibliographic and factual information about the properties of substances in the electronic reference book, the EPIDIF system and Web-lyography, provided in the guidebook of thermal and physical resources.

The electronic reference book on the thermophysical properties of substances is built on the basis of original materials on the thermophysical properties of technically important substances obtained at MEI and ICT RAS in recent years. The layout of the directory was carried out using the Adobe Acrobat technology, which makes it easy to transform the source files prepared by a large team of authors in *.DOC format into PDF files that accumulate all the components of the original document: text, tables, mathematical formulas and figures. The handbook includes detailed data on the thermophysical properties of technically important compounds (water, air components, alkali metals, freons, 
etc.), as well as methodological chapters on general issues of thermal physics, fuels and combustion products. Computed modules are attached to the directory for calculating property tables in the parameter range that the user specifies.

The EPIDIF system is designed to analyze and calculate the transport properties of pure gases and gas mixtures within the framework of the molecular-kinetic theory. The system stores data on the parameters of the three-parameter potential (collision diameter, pit depth, repulsive track steepness index), rotational collision numbers, as well as detailed information on the error of these parameters found during statistical processing of experimental data. The system has its own bibliographic database, which contains information about publications containing experimental data and calculations of transport properties and interaction potentials. The database contains information for both pure gases and mixtures, indicating in each record for which pair of interacting particles there are data on the potentials or collision integrals.As a guide to the network resources "Information Triptych ..." includes a bibliographic database covering various types of thematic resources: portals, electronic periodicals, software, reference books, bibliographic indexes, etc. The entire collection of links is built in a hierarchical structure. Each entry includes, in addition to the URL, a detailed bibliographic description. The system automatically controls the availability of links in the network. All elements of the "Information Triptych ..." are placed on the WEBserver, which provides access for a wide range of users and allows you to take advantage of existing network programming technologies and create dynamic (updated) documents.Information systems that combine the merits of existing codes and eliminate the listed disadvantages will be an important tool for the development of theories of substance properties and phase transformations.

\section{BUILDING A DOMAIN MODEL}

The development of models of information systems and algorithms for the search for functional dependencies in data arrays presupposes, above all, the construction of a domain model. The ontology is usually used as a domain model. However, this concept has a very broad interpretation. First of all, let us clarify exactly what understanding of the term ontology we will adhere to in this work. Earlier, we carried out (as applied to the subject area under consideration) the establishment of certainty in understanding and delimiting the use of the terms "thesaurus" and "ontology". A more or less unambiguous interpretation of the term "thesaurus" was formed in the late 1960s: it is "a dictionary-directory containing all the lexical units of an information retrieval language descriptors (together with keywords that are considered to be synonyms within this information retrieval system these descriptors), and the descriptors in the dictionary should be systematized by meaning, and the semantic links between them are explicitly expressed "[8].As for the term "ontology", nowadays, ontology often has come to mean a wide range of structures representing knowledge about a particular subject area with varying degrees of formalization [9]: 1) a dictionary with definitions; 2) simple taxonomy; 3) thesaurus (taxonomy with terms); 4) a model with an arbitrary set of relations; 5) taxonomy and an arbitrary set of relationships; 6) fully axiomatized theory. We have shown [7, 10] that a thesaurus becomes an ontology when the links between descriptors are not just explicated (as provided in the classical definition of the thesaurus), but are also classified by universal dependencies such as "common-private", "partwhole", "Cause - effect", etc. Of course, this is only the "lower boundary" of the complexity of ontology. To work effectively with facts, it follows that entities related to the subject area are represented not only by their denoting terms, but also by a fairly wide range of attributes, i.e. we are talking about ontology, which has well-known features of the domain model. We have developed a domain model "Experimental and theoretical study of the thermophysical properties and phase transformations of coolants" and on the basis of it built the ontological specification of concepts.As already noted, the study of the properties of thermodynamic systems consisting of a large number of strongly interacting particles near the boundaries of the stability of the phases, involves an expert assessment of the existing array of measurement results with a reasonable choice of recommended values and an assessment of their errors. That is why the most important feature of the developed domain model is that the description of each series of experiments is accompanied by a reference to the source of the data and (or) a detailed description of the installation in which these experiments were performed.

\section{INFORMATION MODEL DESCRIPTION OF A SERIES OF EXPERIMENTS}

The description of a series of experiments includes, first of all, information about the test substance: 1 . The name of the substance (medium_name). 2. Chemical formula of the substance. (medium_formula) 3. Phase state of matter (solid, liquid, gaseous) (phase_name).Next, the measured physical quantity (pq_name) selected from the list is indicated: temperature, pressure, density, molar volume, thermal expansion coefficient, relative elongation, sound velocity, diffusion coefficient, phase transition temperatures, thermal diffusivity, heat capacity, enthalpy, electrical conductivity, magnetic permeability.

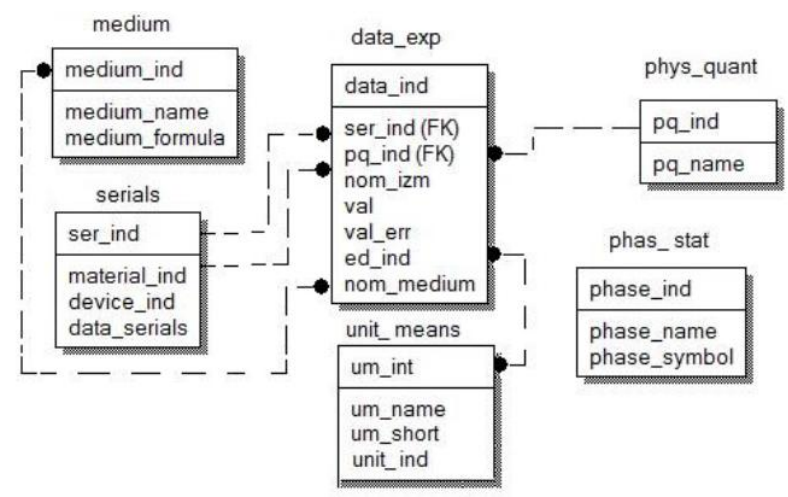

Fig.1. The Structure of the Experimental Data.

For each series of experiments, the following data are given (Fig. 1.): 1. A series of measurements (ser_ind). 2. Number of measurements (nom_izm). 3. The substance (medium_name). 4. Installation (device_ind). 5. System of units (ed_ind). 6. The result of measuring the value (val). 7. Measurement error (val_err).

The indication of the measurement system is necessary because in some cases it may differ from the SI (for example, the temperature is given on the Celsius scale, and not Kelvin). 


\section{RESULTS DISPLAY}

The descriptions of the series of experiments are entered into the PostgreSQL database, after which they can be displayed in a user-friendly form, for example, a graphic one. In fig. 2 shows the features of the behavior of the characteristics of iron in different states. The data are taken from three sources: solid, change in density during melting, liquid.
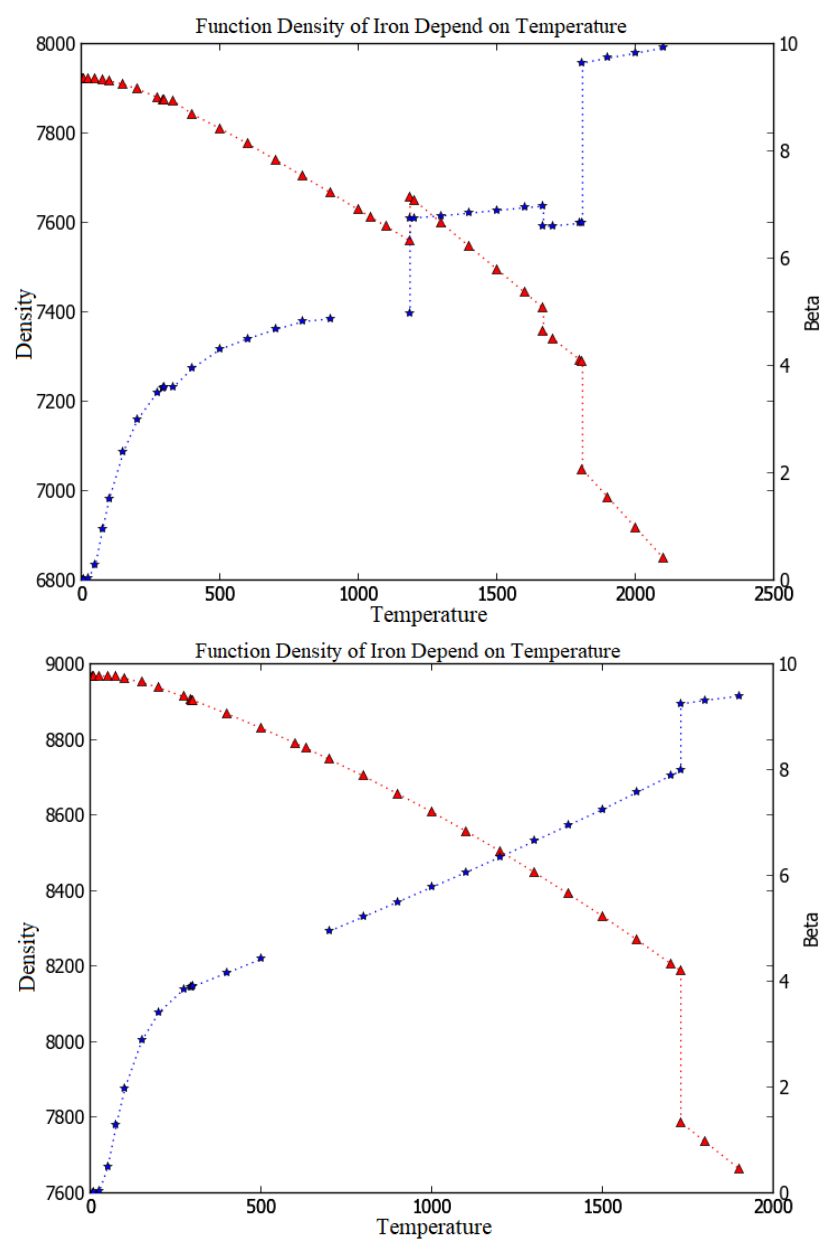

Fig. 2 Temperature Dependence of Density and Coefficient of Volumetric Expansion of Nickel Iron in Solid and Liquid States Density, Kg / M3, Thermal Expansion Coefficient $* 10^{5}, \mathrm{~K}^{-1}$

\section{CONCLUSION}

The paper describes the structure of the created Information and Analytical System "Thermophysical Properties of Substances", designed to work with empirical data on the properties of substances at arbitrary temperatures, pressures and compositions. It can be used to make expert assessments of existing arrays of measurement results and justify the choice of recommended values and the estimation of their errors, the processing of heterogeneous data using physically sound and thermodynamically consistent models. Further work on the system implies further development and extensive use of data mining methods in order to obtain generalizing dependencies for predicting the properties and phase diagrams of new types of unexplored substances.

\section{REFERENCES}

[1] С. В. Станкус, Р. А. Хайрулин, В. Г. Мартынец, Ю. И. Молородов Плотность перфторгексана в окрестности критической точки испарения // ISSS 1818-7994. Вестник НГУ. Серия: Физика. - 2013. Том 8, выпуск 1. - С.73-77.

[2] Barakhnin V.B., Fedotov A.M. Refinement of terminology used in the description of intelligent information systems, based on the semiotic approach // Izvestiya Vuzov. Problems of printing and publishing. 2018. - № 6. - C.73-81.

[3] Zhang, L.; Chen, L.; Liu, J.; Fang, X.; Zhang, Z. Effect of morphology of carbon nanomaterials on thermophysical characteristics, optical properties and photo-thermal conversion performance of nanofluids. Renew. Energy 2016, 99, 888-897.

[4] Azari, A.; Kalbasi, M.; Moazzeni A.; Rahman, A. A Thermal conductivity model for nanofluids heat transfer enhancement. Pet. Sci. Technol. 2014, 32, 91-99.

[5] L. Coco-Enríquez, J. Muñoz-Antón, and J. M. MartínezVal, "New text comparison between $\mathrm{CO} 2$ and other supercritical working fluids (ethane, $\mathrm{Xe}, \mathrm{CH} 4$ and N2) in line- focusing solar power plants coupled to supercritical Brayton power cycles," International Journal of Hydrogen Energy, vol. 42, no. 28, pp. 17611-17631, 2017.

[6] A. G. Memon and R. A. Memon, "Thermodynamic analysis of a trigeneration system proposed for residential application," Energy Conversion and Management, vol. 145, pp. 182-203, 2017. View at Publisher .

[7] C. Yue, D. Han, W. Pu, and W. He, "Parametric analysis of a vehicle power and cooling/heating cogeneration system," Energy, vol. 115, pp. 800-810, 2016.

[8] K. Uebel, P. Rossger, U. Prüfert, A. Richter, and B. Meyer, "A new CO conversion quench reactor design," Fuel Processing Technology, vol. 148, pp. 198-208, 2016.

[9] M. Hashemian, S. Jafarmadar, J. Nasiri, and H. Sadighi Dizaji, "Enhancement of heat transfer rate with structural modification of double pipe heat exchanger by changing cylindrical form of tubes into conical form," Applied Thermal Engineering, vol. 118, pp. 408-417, 2017.

[10] Sura Khalil Ibrahim, Zainab Taha Jebur, "Impact of Information Communication Technology on Business Firms," International Journal of Science and Engineering Applications, Volume 8-Issue 02, PP.53-56, 2019. 